\title{
Comparison between Conventional Ring and Compact Spinning of some Egyptian Cotton Varieties
}

\author{
El-Banna1, M. A., M. A. A. Nassar', M. A. M. Negm², A. A. A. El-Banna1 \\ and A. M. Pharoun ${ }^{2}$ \\ ${ }^{1}$ Plant Production Department - Faculty of Agriculture (Saba-Basha) - \\ Alexandria University. \\ ${ }^{2}$ Cotton Research Institute - Agricultural Research Center, Giza.
}

\begin{abstract}
The current experiments was conducted at Plant Production Department, Faculty of Agriculture (Saba-Basha) Alexandria University, during 2019 season to evaluate the fiber and yarn properties of combed yarns by compact and conventional ring spun yarns for three Egyptian cotton varieties (Giza 94, Giza 92 and Giza 86) which belong to long staple category under three yarn count ( $\mathrm{Ne} \mathrm{80's,} \mathrm{Ne}$ 100's and $\mathrm{Ne} 120$ 's). The fiber properties were measured as well as yarn properties by two spinning system were made at Cotton Research Institute under comparable technological condition on Rstl Marzoli ring and compact spinning fitted with Olfil system on one frame. The results showed that the compact spinning surpassed than conventional ring spinning in yarn traits i.e. lea count strength product (LCSP), single yarn strength (cN/tex), elongation (\%), CV\%, thin and thick places, neps, total imperfections and yarn hairiness. as that the compact spinning recorded the higher desirable values for lea count strength product (LCSP) (2900), single yarn strength (cN/tex) (25.39) and elongation (\%) (5.03). as recorded the lower desirable values for CV\%, thin (16) and thick places (36), neps (68), total imperfections (120) and yarn hairiness (1.60). As well as cotton variety Giza 92 recorded the desirable values for lea count strength product (LCSP) (3030), single yarn strength (cN/tex) (25.24) and CV\% (12.08). On the other side, Giza 86 recorded the better values for thin (11) and thick places (21), neps (67), total imperfections (99) and yarn hairiness (1.80). Yarn count $\mathrm{Ne} 80$ 's gave the desirable values for above studied traits. The first order and second order interaction were significant differences for all studied traits. From the previous results it could be say that cotton variety Giza 92 under both compact spinning and Ne 80's recorded the best values for the studied characters. In general, the properties of combed yarns produced with compact spinning system are better than the those with conventional ring spinning system, compact yarns were having better tensile properties, elongation ratio and lower hairiness than conventional ring yarns, that could be due to the elimination of spinning triangle in spinning system, and the decrease from the flight of fibers of structure yarn increase and this leads to higher yarn tenacity and elongation ratio. Also, Compact spun combed yarn was to found to have higher evenness and lower number of total imperfection values (thin places, thick places and neps). If the evenness property of compact yarn can be improved, it will have a potential for improving quality and profitability of cotton yarn manufacturing.
\end{abstract}

Keywords: Compact spinning, conventional ring spinning, combed yarn, fiber properties, Egyptian cotton varieties, yarn properties.

\section{INTRODUCTION}

Quality and quality management are the key words used in our time; cost and quality are the most important factors for any company. The raw material cost in a spinning mill reaches up to $60 \%$ of the production cost (Staler, 1995 and 2000). Krause and Soliman (1991) and Basal and Oxenham, (2006) show the possibility to use a compact spinning to improve the quality of the yarns produced from short staple cotton fibers in order to get use of the improvement in the compact yarn properties. 
In compact spinning, the "spinning triangle" is eliminated and almost all fibers are incorporated into the yarn structure under the same tension. This leads to significant advantages such as increasing yarn tenacity, yarn abrasion resistance and reducing yarn hairiness (Kampen, 2000 and Cheng and $\mathrm{Yu}$, 2003).

Krifa and Ethridge (2003) and Cheng and Yu (2003) reported that compact spun yarn strength and elongation at break point are higher by about (15\% and $20 \%)$, respectively. Furthermore, the yarn hairiness becomes $(70 \%)$ less, and the coefficient of variation of the yarn was lower by $(15 \%)$ than that for the equivalent ring spun yarn.

Compact spinning is an important alternative for compact yarn production. The system is cheaper and less complicated than pneumatic compact yarn spinning systems. Furthermore, there is not any additional energy consumption during the spinning process Jayavarthanavelu (2006) and Basal and Oxenham (2006) found that high tenacity values of compact yarns can be attributed to the higher rate and amplitude of fiber migration in these yarns compared to those in conventional ring yarns.

The yarns spun on the compact spinning system are characterized by higher tenacity, higher elongation at break, smaller mass irregularity measured at short segments, and significantly lower hairiness than those spun on the conventional ring spinning frame. For the long staple Egyptian cotton varieties, the breaking force or single yarn strength of the compact yarn was $17.63 \%$ higher than the conventional ring spun yarn (El-Sayed and Sanad, 2007).

In conventional ring spinning, zone between the nip line of the delivery rollers and the twisted end of the yarn is called the "spinning triangle". This is the most critical part of the ring spinning system. In this zone, the fiber assembly doesn't have any twist. The edge fibers play out from this zone, and make little or no contribution to the yarn tenacity. Furthermore, they lead to yarn hairiness (Ömeroğlu and Ülkü, 2007).

The reconstructed structure of the ring yarn and the compact yarn segments were compared, and compact yarn presented different structural characteristics, where there are more regular fibers within yarns, and the arrangement of fibers in the cross-section presented a periphery-dense and core-uniform packing. All these findings provide evidence for better understanding of the relatively higher strength and less hairiness of compact yarns (Wu et al., 2009).

The compact spinning yarns have the following advantages when compared to the ring spinning yarns: higher strength and elongation at break, somewhat equivalent or higher yarn unevenness, and a significantly lower number of yarn faults such as thin, thick places and neps, as well as lower hairiness (Sanad et al., 2011). 
The compact yarns have lower hairiness than conventional ring yarns because of the elimination of spinning triangle in spinning system. The compact spinning system slightly increases the evenness and imperfection values of yarns. However, as the yarn becomes finer these effects gradually disappear (Altas and Kadoğlu, 2012).

Iqbal (2018) reported that compact yarns are claimed to be stronger and less hairy dye to the improved fiber binding, and have better yarn elongation and yarn irregularity values compared with conventional ring yarn.

The goal of this work is to make best use of compact spinning taking the advantage of compact yarn structure in processing of combed compact yarns instead of combed ring yarn. A comparative study of the physical and mechanical properties of yarn produced from the three Egyptian cotton varieties on the two spinning systems is studied.

\section{MATERIALS AND METHODS}

In this study used three commercial Egyptian cotton varieties (Giza 94, Giza 92 and Giza 86) long staple belong combed roving during 2019 season. The fiber properties measured with the Uster® HVI 900 testing instrument in accordance with ASTM (1991). D5867-05, were given in Table (1).

Chosen produce three yarn count (Ne 80's, Ne100's and Ne 120's) count yarns spun at twist multipliers 4.0. The yarns were produced in both compact and conventional ring spinning. A standard spinning preparation and modern machinery in the experimental spinning mill, Cotton Research Institute, were used to produce conventional and compact carded yarns under comparable technological conditions on the RST1 Marzoli ring and compact spinning fitted with (Olfil System) on one frame, as shown in Figure (1).

After the spinning trials, the physical properties of each yarn sample were measured according to ASTM (1991). D-2256-80, D-1425-81 and the measurement results of conventional ring yarns and compact yarns were compared to each other. Yarn evenness (CV\%), hairiness and imperfections values were measured on Uster Tester 3 (the measurement length was 400 $\mathrm{m} /$ bobbin). Yarn tenacity (cN/Tex) and elongation at break (\%) were measured on a Statimat ME with 120 breaks per sample.

All tests were performed after the yarns were kept in standard atmospheric conditions for 24 hours ( $65 \pm 2 \%$ relative humidity, $20 \pm 2^{\circ} \mathrm{C}$ ).

In the analysis of test results, factorial ANOVA and multiple ANOVA (L.S.D.) methods were designed as analysis of variance experiment with three replications at 0.05 significance level. 
Table (1). Fiber properties of the commercial Egyptian cotton varieties during 2019 season

\begin{tabular}{|c|c|c|c|c|}
\hline \multicolumn{2}{|l|}{ Characters } & Giza 94 & Giza 92 & Giza 86 \\
\hline \multirow{2}{*}{\multicolumn{2}{|c|}{$\begin{array}{l}\text { Micronair reading } \\
\text { Maturity ratio (\%) }\end{array}$}} & 3.91 & 3.70 & 4.44 \\
\hline & & 92 & 90 & 91 \\
\hline \multirow{3}{*}{ Fiber length } & U.H.M.L (mm) & 34.72 & 34.40 & 32.87 \\
\hline & Uniformity index (\%) & 88.7 & 87.2 & 86.5 \\
\hline & Short fiber index (\%) & 6.03 & 5.73 & 6.07 \\
\hline \multirow{2}{*}{$\begin{array}{l}\text { Strength \& } \\
\text { Elongation \% }\end{array}$} & Fiber strength (g/tex) & 44.30 & 48.63 & 45.50 \\
\hline & Fiber elongation (\%) & 5.77 & 5.80 & 5.90 \\
\hline \multirow{2}{*}{$\begin{array}{l}\text { Values of color } \\
\text { attributes }\end{array}$} & Reflectance degree (Rd \%) & 77.49 & 77.37 & 74.39 \\
\hline & Yellowness degree $(+b)$ & 9.00 & 8.65 & 8.95 \\
\hline \multirow{2}{*}{ Fiber trash } & Trash count (TC) & 23 & 38 & 35 \\
\hline & Trash area (TA) & 0.50 & 0.65 & 0.63 \\
\hline
\end{tabular}

U.H.M.L.: upper half means length.

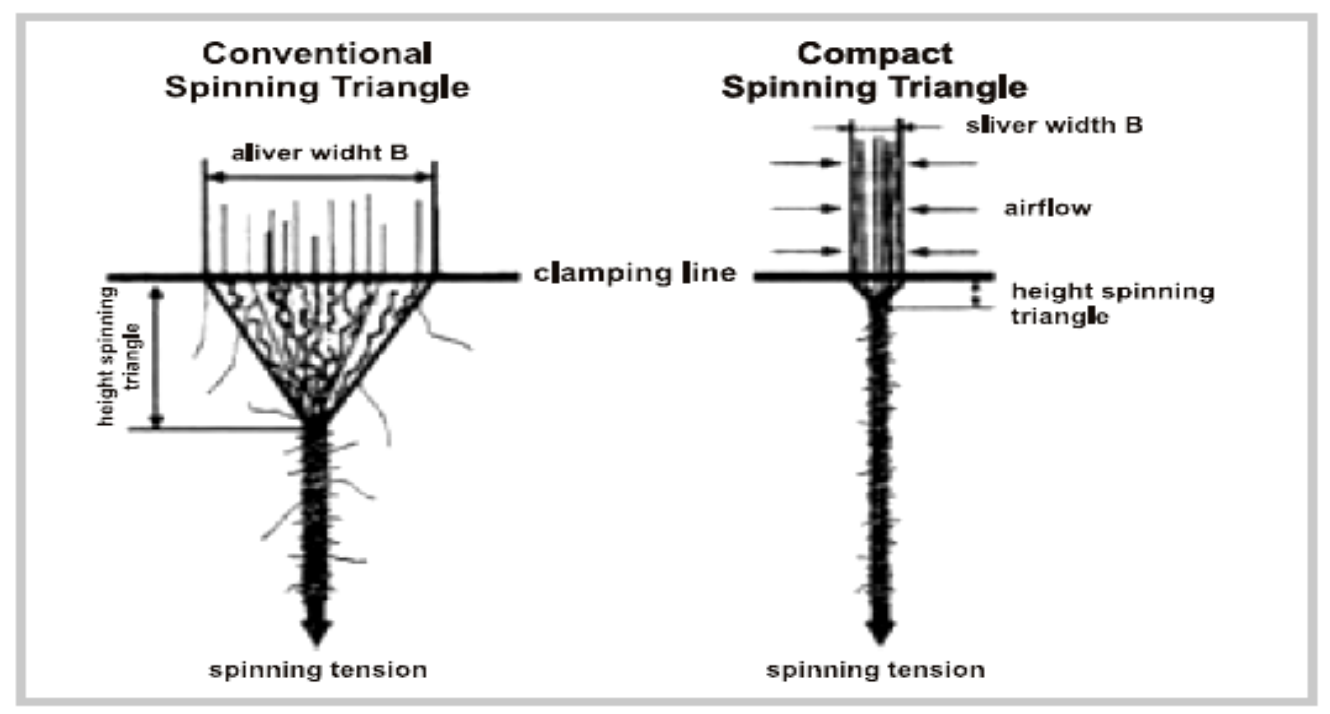

Figure (1). Yarn formation in conventional and compact ring spinning. Artzt, (2000)

\section{RESULTS AND DISCUSSION}

\section{Evaluation of yarn properties}

According to the experimental results of yarn properties, compact yarns were better quality than conventional yarns due to elimination of fiber fly in compact yarn spinning system. The main effects of spinning system, cotton varieties and yarn count on yarn properties as in Table (2), have been analyzed also the interaction effects of spinning system and cotton varieties $(S \times V)$, spinning system and yarn count $(\mathrm{S} \times \mathrm{C})$, cotton varieties and yarn count $(\mathrm{V} \times \mathrm{C})$ and also spinning system, cotton varieties and yarn count $(S \times V \times C)$ as in Tables (3 and 4). 
Presented results in Table (2) showed that spinning systems, cotton varieties and yarn count besides their first and second order interactions had significant effects on all the studied traits as follows.

Based on the analysis results following conclusions can be drawn:

\section{Yarn tenacity and elongation ratio results:}

The results presented for tenacity measurement of conventional and compact spun yarns, according to statistical analysis showed that the effect of spinning system has significant effect on all cotton varieties for lea count strength product (LCSP), single yarn strength (cN/tex) and elongation ratio as shown in Tables (2 and 3 ). The tenacity and elongation values of combed compact yarns are significantly higher than combed conventional ring yarns.

The interactions between spinning system and cotton varieties $(\mathrm{S} \times \mathrm{V})$ are shown in Figure (2) and Table (3). The results indicate that Giza 92 had the highest tenacity followed by Giza 94, and Giza 86, respectively. Also the interaction between spinning system and yarn count $(S \times C)$ are shown in Figure (2) and Table (3) is effect statistically significant on both combed yarn tenacity and elongation ratio. The tenacity and elongation values of conventional and compact spun yarns increase as the yarn becomes coarser.

Table (3) and Figure (2) compares the results of yarn strength obtained on the yarn count ( $\mathrm{Ne} 80$ 's, Ne 100's, and Ne 120's) both combed compact yarns and combed conventional ring yarns from the cotton varieties. The results showed that the yarn strength decreased with the increase of the yarn count, and that the most decrease in yarn strength with increasing yarn count recorded for Giza 94, variety and the lowest variety in decrease in the yarn strength was the Giza 86. 
Table (2). Effect of spinning system (S), cotton variety (V), yarn count (C) and their interactions on yarn properties during 2019 season

\begin{tabular}{|c|c|c|c|c|c|c|c|c|c|c|}
\hline \multirow{2}{*}{ Entries } & \multicolumn{3}{|c|}{ Strength \& Elongation (\%) } & \multicolumn{2}{|c|}{ Evenness } & \multicolumn{4}{|c|}{ Imperfection } & \multirow[b]{2}{*}{ Hairiness } \\
\hline & LCSP & $\begin{array}{l}\text { Strength } \\
\text { (cN/tex) }\end{array}$ & $\begin{array}{c}\text { Elongation } \\
(\%)\end{array}$ & $\mathbf{U}(\%)$ & C.V.(\%) & $\begin{array}{c}\text { Thin } \\
\text { places } \\
(-50 \%)\end{array}$ & $\begin{array}{c}\text { Thick } \\
\text { places } \\
(+50 \%)\end{array}$ & $\begin{array}{c}\text { Neps } \\
(+200 \%)\end{array}$ & $\begin{array}{c}\text { Total } \\
\text { imperfection }\end{array}$ & \\
\hline \multicolumn{11}{|c|}{ Spinning system (S) } \\
\hline Ring spinning & $2775 b$ & $24.51 b$ & $4.81 b$ & $10.78 \mathrm{a}$ & $13.17 \mathrm{a}$ & $21 \mathrm{a}$ & $44 \mathrm{a}$ & $74 \mathrm{a}$ & $139 a$ & $2.45 \mathrm{a}$ \\
\hline $\begin{array}{c}\text { Compact spinning } \\
\text { L.S.D }\end{array}$ & $\begin{array}{c}2900 \mathrm{a} \\
29\end{array}$ & $\begin{array}{c}25.39 \mathrm{a} \\
0.22\end{array}$ & $\begin{array}{c}5.03 \mathrm{a} \\
0.12\end{array}$ & $\begin{array}{c}9.88 b \\
0.13\end{array}$ & $\begin{array}{c}12.18 b \\
0.16\end{array}$ & $\begin{array}{c}16 b \\
2\end{array}$ & $\begin{array}{c}36 b \\
3\end{array}$ & $\begin{array}{c}68 b \\
3\end{array}$ & $\begin{array}{c}120 b \\
6\end{array}$ & $\begin{array}{l}1.60 \mathrm{~b} \\
\mathbf{0 . 0 5}\end{array}$ \\
\hline \multicolumn{11}{|c|}{ Cotton varieties (V) } \\
\hline Giza 94 & $2790 b$ & $24.97 b$ & $4.55 \mathrm{c}$ & $10.91 \mathrm{a}$ & $13.37 \mathrm{a}$ & $24 a$ & $54 \mathrm{a}$ & $76 \mathrm{a}$ & $154 \mathrm{a}$ & $2.05 \mathrm{~b}$ \\
\hline Giza 92 & $3030 \mathrm{a}$ & $25.24 \mathrm{a}$ & $4.83 \mathrm{~b}$ & $9.95 \mathrm{c}$ & $12.08 \mathrm{c}$ & $21 b$ & $46 \mathrm{~b}$ & $68 \mathrm{~b}$ & $135 b$ & $2.24 \mathrm{a}$ \\
\hline Giza 86 & $2695 \mathrm{c}$ & $24.65 \mathrm{c}$ & $5.52 \mathrm{a}$ & $10.13 b$ & $12.57 \mathrm{~b}$ & $11 \mathrm{c}$ & $21 \mathrm{c}$ & $67 \mathrm{c}$ & $99 \mathrm{c}$ & $1.80 \mathrm{c}$ \\
\hline L.S.D & 36 & 0.28 & 0.15 & 0.16 & 0.21 & 3 & 4 & 4 & 7 & 0.06 \\
\hline \multicolumn{11}{|c|}{ Yarn count (C) } \\
\hline 80's & $3030 a$ & $25.85 \mathrm{a}$ & $5.18 \mathrm{a}$ & $9.66 \mathrm{c}$ & $11.75 \mathrm{c}$ & $8 \mathrm{c}$ & $25 \mathrm{c}$ & $60 \mathrm{c}$ & $93 \mathrm{c}$ & $2.23 \mathrm{a}$ \\
\hline 100's & $2845 b$ & $25.31 b$ & $4.97 \mathrm{~b}$ & $10.37 b$ & $12.56 \mathrm{~b}$ & $18 b$ & $42 \mathrm{~b}$ & $62 \mathrm{~b}$ & $122 b$ & $1.99 \mathrm{~b}$ \\
\hline 120 's & $2640 \mathrm{c}$ & $23.68 \mathrm{c}$ & $4.77 \mathrm{c}$ & $10.97 \mathrm{a}$ & $13.72 \mathrm{a}$ & $30 \mathrm{a}$ & $54 \mathrm{a}$ & $90 \mathrm{a}$ & $174 a$ & $1.86 \mathrm{c}$ \\
\hline L.S.D & 36 & 0.28 & 0.15 & 0.16 & 0.21 & 3 & 4 & 4 & 7 & 0.06 \\
\hline \multicolumn{11}{|c|}{ Interaction } \\
\hline S X V & * & * & * & * & * & * & * & * & * & * \\
\hline$S \times C$ & * & * & * & * & * & * & * & * & * & * \\
\hline $\mathrm{V} \times \mathrm{C}$ & * & * & * & * & * & * & * & * & * & * \\
\hline$S \times V \times C$ & * & * & * & * & * & * & * & * & * & * \\
\hline
\end{tabular}

LCSP: lea count strength product - U\%: percent mean deviation - C.V. \%: coefficient of variation.

* Significant difference at 0.05 level of probability.

Means within each column with the same letters are not significant difference at 0.05 level of probability. 
Table (3). The first order interactions spinning system $x$ cotton variety (S $x$ V), spinning system $x$ yarn count (S $x$ C) and cotton varieties $x$ yarn count $(V \times C)$ for yarn properties during 2019 season

\begin{tabular}{|c|c|c|c|c|c|c|c|c|c|c|c|}
\hline \multirow{2}{*}{\multicolumn{2}{|c|}{$\gamma_{\text {Entries }}^{\text {Traits }}$}} & \multicolumn{3}{|c|}{ Strength \& Elongation (\%) } & \multicolumn{2}{|c|}{ Evenness } & \multicolumn{4}{|c|}{ Imperfection } & \multirow[b]{2}{*}{ Hairiness } \\
\hline & & LCSP & $\begin{array}{l}\text { Strength } \\
\text { (cN/tex) }\end{array}$ & $\begin{array}{c}\text { Elongation } \\
(\%)\end{array}$ & $U(\%)$ & C.V.(\%) & $\begin{array}{c}\text { Thin places } \\
(-50 \%)\end{array}$ & $\begin{array}{c}\text { Thick places } \\
(+50 \%)\end{array}$ & $\begin{array}{c}\text { Neps } \\
(+200 \%)\end{array}$ & $\begin{array}{c}\text { Total } \\
\text { imperfection }\end{array}$ & \\
\hline \multicolumn{12}{|c|}{ Spinning system $(\mathrm{S}) \times$ cotton varieties $(\mathrm{V})$} \\
\hline \multirow{3}{*}{$\begin{array}{c}\text { Ring } \\
\text { spinning }\end{array}$} & G 94 & $2730 \mathrm{~d}$ & $24.55 \mathrm{de}$ & $4.51 \mathrm{e}$ & $11.38 \mathrm{a}$ & $13.89 \mathrm{a}$ & $28 \mathrm{a}$ & $58 a$ & $81 \mathrm{a}$ & $167 \mathrm{a}$ & $2.47 \mathrm{~b}$ \\
\hline & G 92 & $2965 \mathrm{~b}$ & $24.82 \mathrm{~cd}$ & $4.69 \mathrm{de}$ & $10.37 \mathrm{~b}$ & $12.54 \mathrm{c}$ & $24 a b$ & $50 \mathrm{~b}$ & $71 \mathrm{~b}$ & $145 \mathrm{~b}$ & $2.67 \mathrm{a}$ \\
\hline & G 86 & $2630 \mathrm{e}$ & $24.15 \mathrm{e}$ & $5.46 \mathrm{~b}$ & $10.60 \mathrm{~b}$ & $13.09 \mathrm{~b}$ & $12 \mathrm{c}$ & $24 d$ & $70 \mathrm{~b}$ & $106 \mathrm{~d}$ & $2.21 \mathrm{c}$ \\
\hline \multirow{3}{*}{$\begin{array}{l}\text { Compact } \\
\text { spinning }\end{array}$} & G 94 & $2855 \mathrm{c}$ & $25.38 \mathrm{ab}$ & $4.81 \mathrm{~cd}$ & $10.45 b$ & $12.86 \mathrm{~b}$ & $20 \mathrm{~b}$ & $49 \mathrm{~b}$ & $72 \mathrm{~b}$ & $141 \mathrm{~b}$ & $1.62 \mathrm{e}$ \\
\hline & G 92 & 3090 a & $25.65 \mathrm{a}$ & $4.97 \mathrm{C}$ & $9.53 \mathrm{c}$ & $11.62 \mathrm{e}$ & $18 \mathrm{~b}$ & $42 \mathrm{c}$ & $66 \mathrm{~b}$ & $126 \mathrm{c}$ & $1.80 \mathrm{~d}$ \\
\hline \multirow{2}{*}{\multicolumn{2}{|c|}{ L.S.D G 86}} & $2760 d$ & $25.14 \mathrm{bc}$ & $5.79 a$ & $9.66 \mathrm{c}$ & $12.06 \mathrm{~d}$ & $9 \mathrm{c}$ & $17 e$ & $65 \mathrm{~b}$ & $91 \mathrm{e}$ & $1.39 \mathrm{f}$ \\
\hline & & 55 & 0.43 & 0.23 & 0.25 & 0.31 & 5 & 6 & 6 & 11 & 0.09 \\
\hline \multicolumn{12}{|c|}{ Spinning system $(\mathbf{S}) \times$ yarn count $(\mathrm{C})$} \\
\hline \multirow{3}{*}{$\begin{array}{l}\text { Ring } \\
\text { spinning }\end{array}$} & 80's & $2960 \mathrm{~b}$ & $25.39 \mathrm{c}$ & $5.14 \mathrm{a}$ & $10.10 \mathrm{~d}$ & $12.23 \mathrm{c}$ & $10 \mathrm{~d}$ & $28 d$ & $61 \mathrm{c}$ & $99 \mathrm{e}$ & $2.65 \mathrm{a}$ \\
\hline & 100 's & $2790 \mathrm{~d}$ & $24.85 \mathrm{~d}$ & $4.91 \mathrm{~b}$ & $10.83 \mathrm{~b}$ & $13.06 \mathrm{~b}$ & $21 \mathrm{bc}$ & $44 \mathrm{bc}$ & $65 c$ & $130 c$ & $2.40 \mathrm{~b}$ \\
\hline & 120 's & $2580 \mathrm{f}$ & $23.29 \mathrm{f}$ & $4.71 \mathrm{~b}$ & $11.42 \mathrm{a}$ & $14.22 \mathrm{a}$ & $33 \mathrm{a}$ & $60 \mathrm{a}$ & $95 \mathrm{a}$ & $188 \mathrm{a}$ & $2.30 \mathrm{c}$ \\
\hline \multirow{3}{*}{$\begin{array}{l}\text { Compact } \\
\text { spinning }\end{array}$} & 80 's & $3105 a$ & $26.32 \mathrm{a}$ & $5.22 \mathrm{a}$ & $9.22 \mathrm{e}$ & $11.26 \mathrm{~d}$ & $6 d$ & $22 d$ & $58 \mathrm{c}$ & $86 f$ & $1.80 \mathrm{~d}$ \\
\hline & 100 's & $2900 \mathrm{c}$ & $25.77 \mathrm{bc}$ & $5.02 \mathrm{a}$ & $9.91 \mathrm{~d}$ & $12.05 \mathrm{c}$ & $16 \mathrm{c}$ & $39 \mathrm{c}$ & $59 \mathrm{c}$ & $114 \mathrm{~d}$ & $1.58 \mathrm{e}$ \\
\hline \multirow{2}{*}{\multicolumn{2}{|c|}{ L.S.D }} & $2700 \mathrm{e}$ & $24.07 \mathrm{e}$ & $4.83 \mathrm{~b}$ & $10.51 \mathrm{~d}$ & $13.22 b$ & $26 \mathrm{~b}$ & $48 \mathrm{~b}$ & $86 \mathrm{~b}$ & $160 \mathrm{~b}$ & $1.43 \mathrm{f}$ \\
\hline & & 55 & 0.43 & 0.23 & 0.25 & 0.31 & 5 & 6 & 6 & 11 & 0.09 \\
\hline \multicolumn{12}{|c|}{ Cotton varieties $(\mathrm{V}) \times$ yarn count $(\mathrm{C})$} \\
\hline \multirow{4}{*}{ Giza 94} & 80 's & $3030 \mathrm{~b}$ & $25.74 b$ & $4.98 \mathrm{~b}$ & $10.13 \mathrm{~d}$ & $12.30 \mathrm{c}$ & $8 d$ & $31 \mathrm{~d}$ & $73 \mathrm{~b}$ & $112 \mathrm{~d}$ & $2.21 \mathrm{~b}$ \\
\hline & 100 's & $2780 \mathrm{c}$ & $25.72 b$ & $4.42 \mathrm{~cd}$ & $11.04 \mathrm{~b}$ & $13.50 \mathrm{~b}$ & $28 \mathrm{~b}$ & $60 \mathrm{~b}$ & $76 \mathrm{~b}$ & $164 \mathrm{~b}$ & $2.04 \mathrm{c}$ \\
\hline & 120's & 2565 e & $23.43 \mathrm{~d}$ & $4.25 \mathrm{~d}$ & $11.56 \mathrm{a}$ & $14.31 \mathrm{a}$ & $37 \mathrm{a}$ & $69 a$ & $79 \mathrm{~b}$ & $185 a$ & $1.89 \mathrm{~d}$ \\
\hline & 80 's & $3255 \mathrm{a}$ & $26.43 \mathrm{a}$ & $5.06 \mathrm{~b}$ & $9.28 \mathrm{e}$ & $11.13 \mathrm{~d}$ & $15 \mathrm{c}$ & $30 d$ & $67 \mathrm{~b}$ & $112 \mathrm{~d}$ & $2.54 \mathrm{a}$ \\
\hline \multirow[t]{3}{*}{ Giza 92} & 100's & $3060 \mathrm{~b}$ & $25.53 b$ & $4.81 \mathrm{bc}$ & $9.99 \mathrm{~d}$ & $12.03 \mathrm{c}$ & $19 \mathrm{c}$ & $46 \mathrm{c}$ & $68 \mathrm{~b}$ & $133 c$ & $2.13 b c$ \\
\hline & 120 's & $2770 \mathrm{c}$ & $23.73 \mathrm{~d}$ & $4.64 \mathrm{c}$ & $10.57 \mathrm{c}$ & $13.08 \mathrm{~b}$ & $28 \mathrm{~b}$ & $62 a b$ & $72 \mathrm{~b}$ & $162 \mathrm{~b}$ & $2.04 \mathrm{c}$ \\
\hline & 80 's & $2810 \mathrm{c}$ & $25.39 \mathrm{~b}$ & $5.51 \mathrm{a}$ & $9.55 \mathrm{e}$ & $11.81 \mathrm{c}$ & $2 d$ & $13 \mathrm{e}$ & $39 \mathrm{c}$ & $54 \mathrm{e}$ & $1.93 \mathrm{~cd}$ \\
\hline Giza 86 & 100 's & $2695 d$ & $24.67 \mathrm{c}$ & $5.67 \mathrm{a}$ & $10.08 d$ & $12.13 \mathrm{c}$ & $7 d$ & $18 \mathrm{e}$ & $42 \mathrm{c}$ & $67 \mathrm{e}$ & $1.80 \mathrm{de}$ \\
\hline \multicolumn{2}{|c|}{ L.S.D ${ }^{120 ' s}$} & $\begin{array}{c}2585 \text { e } \\
74\end{array}$ & $\begin{array}{c}23.88 \mathrm{~d} \\
0.57\end{array}$ & $\begin{array}{c}5.41 \mathrm{a} \\
0.31\end{array}$ & $\begin{array}{c}10.77 b c \\
0.33\end{array}$ & $\begin{array}{c}13.77 b \\
0.42\end{array}$ & $\begin{array}{c}24 \mathrm{bc} \\
6\end{array}$ & $\begin{array}{c}30 \mathrm{~d} \\
8\end{array}$ & $\begin{array}{c}120 \mathrm{a} \\
8\end{array}$ & $\begin{array}{c}174 a b \\
15\end{array}$ & $\begin{array}{c}1.67 \mathrm{e} \\
\mathbf{0 . 1 3}\end{array}$ \\
\hline
\end{tabular}

LCSP: lea count strength product - U\%: percent mean deviation - C.V. \%: coefficient of variation.

Means within each column with the same letters are not significant difference at 0.05 level of probability. 
Table (4). Effect of spinning system (S), cotton variety (V) and yarn count (C) interactions on yarn properties during 2019 season

\begin{tabular}{|c|c|c|c|c|c|c|c|c|c|c|c|c|}
\hline \multicolumn{3}{|c|}{ Traits } & \multicolumn{3}{|c|}{ Strength \& Elongation (\%) } & \multicolumn{2}{|c|}{ Evenness } & \multicolumn{4}{|c|}{ Imperfection } & \multirow[b]{2}{*}{ Hairiness } \\
\hline $\begin{array}{l}\text { Spinning } \\
\text { system } \\
\text { (S) }\end{array}$ & $\begin{array}{l}\text { Varieties } \\
\text { (V) }\end{array}$ & $\begin{array}{c}\text { Yarn } \\
\text { count } \\
\text { (C) }\end{array}$ & LCSP & $\begin{array}{l}\text { Strength } \\
\text { (cN/tex) }\end{array}$ & $\begin{array}{c}\text { Elongation } \\
(\%)\end{array}$ & U (\%) & C.V.(\%) & $\begin{array}{l}\text { Thin } \\
\text { places } \\
(-50 \%)\end{array}$ & $\begin{array}{c}\text { Thick } \\
\text { places } \\
(+50 \%) \\
\end{array}$ & $\begin{array}{c}\text { Neps } \\
(+200 \%)\end{array}$ & $\begin{array}{c}\text { Total } \\
\text { imperfection }\end{array}$ & \\
\hline \multirow{9}{*}{$\begin{array}{c}\text { Ring } \\
\text { spinning }\end{array}$} & \multirow{3}{*}{ Giza 94} & 80 's & $2965 \mathrm{de}$ & $25.35 \mathrm{~b}$ & $4.95 \mathrm{~b}$ & $10.57 \mathrm{c}$ & $12.81 \mathrm{~d}$ & $11 \mathrm{~cd}$ & $36 \mathrm{~cd}$ & $77 \mathrm{~b}$ & $124 \mathrm{~d}$ & $2.64 \mathrm{~b}$ \\
\hline & & 100's & $2720 \mathrm{f}$ & $25.29 \mathrm{~b}$ & $4.38 \mathrm{c}$ & $11.53 \mathrm{ab}$ & $14.00 \mathrm{~b}$ & $32 a b$ & $63 a b$ & $81 \mathrm{~b}$ & $176 a b$ & $2.45 b c$ \\
\hline & & 120 's & $2500 \mathrm{~h}$ & $23.03 \mathrm{e}$ & $4.18 \mathrm{~d}$ & $12.02 \mathrm{a}$ & $14.85 \mathrm{a}$ & $42 \mathrm{a}$ & $76 a$ & $84 \mathrm{~b}$ & $202 a$ & $2.33 \mathrm{~cd}$ \\
\hline & \multirow{3}{*}{ Giza 92} & 80 's & $3180 \mathrm{bc}$ & $25.97 \mathrm{a}$ & $5.00 \mathrm{~b}$ & $9.69 \mathrm{e}$ & $11.62 \mathrm{f}$ & $18 \mathrm{c}$ & $33 d$ & $67 c$ & $118 \mathrm{de}$ & $2.98 \mathrm{a}$ \\
\hline & & 100 's & $3005 d$ & $25.12 \mathrm{bc}$ & $4.77 \mathrm{bc}$ & $10.43 \mathrm{~cd}$ & $12.47 \mathrm{e}$ & $22 \mathrm{bc}$ & $50 \mathrm{bc}$ & $70 \mathrm{bc}$ & $142 \mathrm{~cd}$ & $2.57 \mathrm{~b}$ \\
\hline & & 120 's & $2710 \mathrm{fg}$ & $23.65 d$ & $4.59 \mathrm{c}$ & $11.00 \mathrm{bc}$ & $13.51 \mathrm{c}$ & $32 a b$ & $68 \mathrm{a}$ & $76 \mathrm{~b}$ & $176 a b$ & $2.47 \mathrm{~b}$ \\
\hline & \multirow{3}{*}{ Giza 86} & 80 's & $2735 \mathrm{f}$ & $24.84 \mathrm{c}$ & $5.47 \mathrm{a}$ & $10.04 \mathrm{de}$ & 12.26 ef & $2 d$ & $15 \mathrm{e}$ & $40 d$ & $57 f$ & $2.33 \mathrm{~cd}$ \\
\hline & & 100's & $2640 \mathrm{~g}$ & $24.14 \mathrm{c}$ & $5.59 a$ & $10.52 \mathrm{c}$ & $12.71 \mathrm{~d}$ & $8 d$ & $21 \mathrm{e}$ & $44 \mathrm{~d}$ & $73 \mathrm{f}$ & $2.19 \mathrm{de}$ \\
\hline & & 120 's & $2520 \mathrm{~h}$ & $23.48 \mathrm{de}$ & $5.33 \mathrm{ab}$ & $11.24 \mathrm{~b}$ & $14.29 a b$ & $26 \mathrm{~b}$ & $35 d$ & $124 a$ & $185 a$ & $2.09 \mathrm{e}$ \\
\hline \multirow{10}{*}{$\begin{array}{l}\text { Compact } \\
\text { spinning }\end{array}$} & \multirow{3}{*}{ Giza 94} & 80 's & $3095 \mathrm{~cd}$ & $26.14 \mathrm{a}$ & $5.02 \mathrm{~b}$ & $9.70 \mathrm{e}$ & $11.79 \mathrm{f}$ & $6 \mathrm{~d}$ & $26 \mathrm{~d}$ & $70 \mathrm{bc}$ & $102 \mathrm{e}$ & $1.79 \mathrm{f}$ \\
\hline & & 100 's & $2835 \mathrm{e}$ & $26.07 \mathrm{a}$ & $4.46 \mathrm{c}$ & $10.54 \mathrm{c}$ & $12.99 \mathrm{~d}$ & $24 \mathrm{~b}$ & $59 \mathrm{~b}$ & $71 \mathrm{~b}$ & $154 \mathrm{bc}$ & $1.62 \mathrm{f}$ \\
\hline & & 120 's & $2630 \mathrm{gh}$ & $23.85 d$ & $4.33 \mathrm{~cd}$ & $11.10 \mathrm{~b}$ & $13.77 \mathrm{bc}$ & $33 a$ & $63 a b$ & $75 \mathrm{~b}$ & $171 \mathrm{~b}$ & $1.44 \mathrm{~g}$ \\
\hline & \multirow{3}{*}{ Giza 92} & 80 's & $3330 \mathrm{a}$ & $26.88 \mathrm{a}$ & $5.10 \mathrm{~b}$ & $8.88 \mathrm{f}$ & $10.64 \mathrm{~g}$ & $13 \mathrm{c}$ & $28 d$ & $67 c$ & $108 \mathrm{e}$ & $2.09 \mathrm{e}$ \\
\hline & & 100 's & $3115 c$ & $25.96 \mathrm{a}$ & $4.86 \mathrm{~b}$ & 9.56 ef & $11.58 \mathrm{f}$ & $17 \mathrm{c}$ & $42 \mathrm{c}$ & $65 c$ & $124 d$ & $1.70 \mathrm{f}$ \\
\hline & & 120 's & 2830 ef & $24.11 \mathrm{~cd}$ & $4.77 \mathrm{bc}$ & $10.15 d$ & $12.63 \mathrm{de}$ & $24 \mathrm{~b}$ & $56 \mathrm{~b}$ & $67 c$ & $147 \mathrm{c}$ & $1.59 \mathrm{fg}$ \\
\hline & & 80 's & $2880 \mathrm{e}$ & $25.95 \mathrm{ab}$ & $5.54 \mathrm{a}$ & $9.07 f$ & $11.35 \mathrm{~g}$ & $2 d$ & $12 \mathrm{e}$ & $38 d$ & $52 \mathrm{f}$ & $1.52 \mathrm{~g}$ \\
\hline & \multirow[t]{2}{*}{ Giza 86} & 100 's & $2745 \mathrm{f}$ & $25.20 \mathrm{~b}$ & $5.74 \mathrm{a}$ & $9.63 \mathrm{e}$ & $11.56 f g$ & $6 d$ & $16 \mathrm{e}$ & $40 d$ & $62 f$ & $1.41 \mathrm{gh}$ \\
\hline & & 120 's & $2650 \mathrm{~g}$ & 24.28 c & $5.49 a$ & $10.30 \mathrm{~d}$ & $13.25 \mathrm{~cd}$ & $22 b c$ & $24 \mathrm{de}$ & $116 a$ & $162 \mathrm{~b}$ & $1.24 \mathrm{~h}$ \\
\hline & L.S.D & & 135 & 1.04 & 0.56 & 0.61 & 0.76 & 12 & 14 & 15 & 26 & 0.23 \\
\hline
\end{tabular}

LCSP: lea count strength product - U\%: percent mean deviation - C.V. \%: coefficient of variation. 
According to Table (4) and Figure (2) the results present the effects significant and hold true for both effect spinning system, cotton varieties and yarn count interaction, $(\mathrm{S} \times \mathrm{V} \times \mathrm{C}$ ) on lea count strength product (LCSP), single yarn strength (cN/tex) and elongation ratio. It clearly shows that the compact spinning resulted in a generally higher strength and greater elongation for cotton varieties under study.

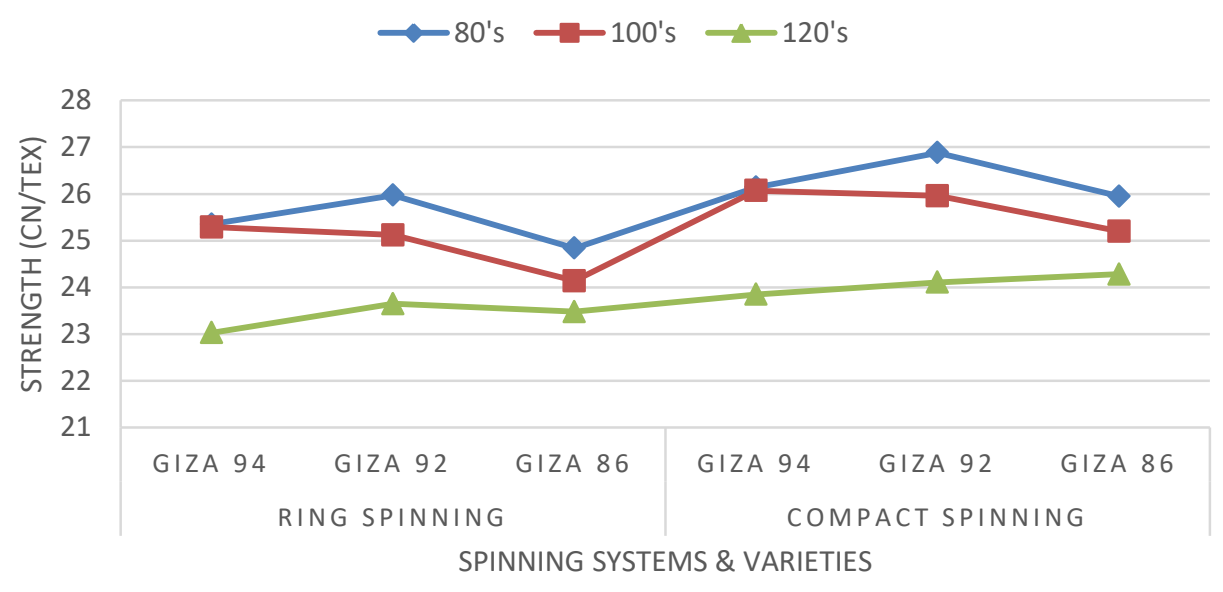

Figure (2). Single yarn strength (cN/tex) as affected by spinning systems, cotton varieties and yarn count interaction

\section{Yarn evenness results:}

The results of conventional and compact spun yarns evenness, according to statistical analysis, that the spinning system its effect on combed yarn evenness as shown in Table (2 and 3 ). Combed compact yarns have higher evenness (less values for $\mathrm{U} \%$ and $\mathrm{CV} \%$ ) than combed conventional ring yarns.

The interaction effect of spinning system and yarn count $(S \times C)$ is statistically significant. The evenness values of conventional and compact spun combed yarns increase as the yarn becomes coarser as shown in Table (3).

According to Table (3) the results present that the effect significant and hold true for both cotton varieties and yarn count interaction ( $\mathrm{V} \times \mathrm{C}$ ). It clearly shows that the Giza 92 variety resulted in a generally higher evenness values for cotton varieties under study.

Analysis of variance of the interaction between spinning system, cotton varieties and yarn count $(\mathrm{S} \times \mathrm{V} \times \mathrm{C}$ ) for yarn evenness data is summarized in Table (4) and Figure (3). Where there is difference in the evenness data, the results indicate that, there is a significant effect of compact spinning on yarn evenness properties although a slight difference in the evenness data. The results are similar for all yarn count. 


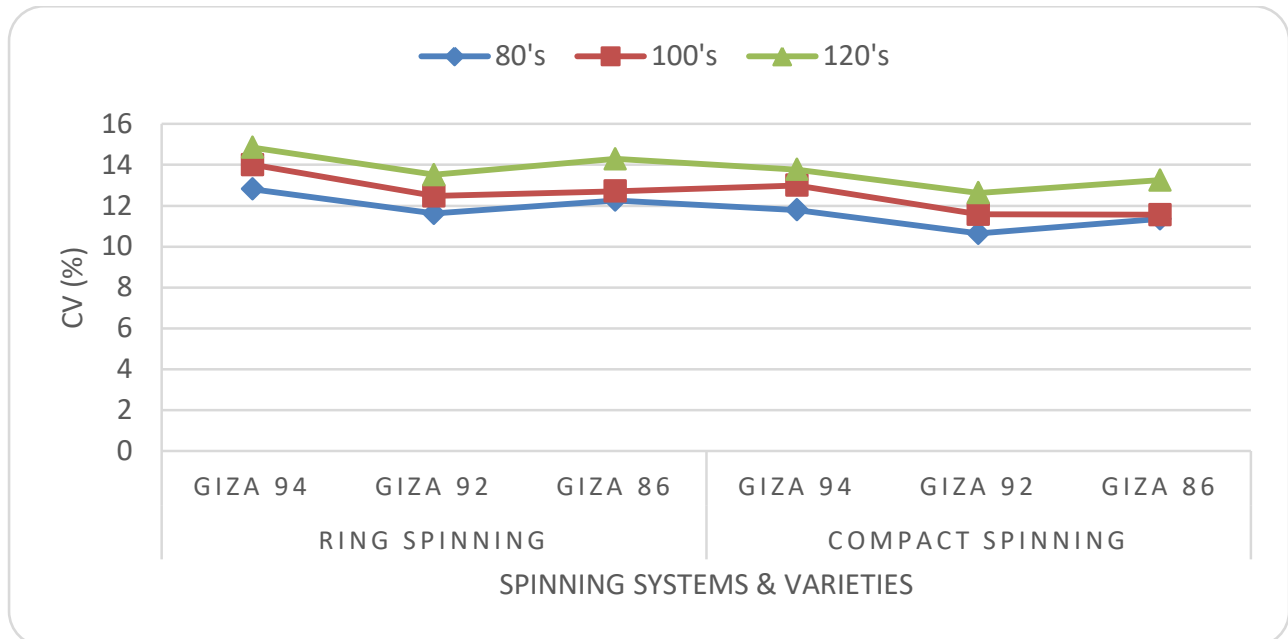

Figure (3). yarn evenness (C.V. \%) as affected by spinning systems, cotton varieties and yarn count interaction

\section{Yarn imperfection results:}

In combed yarns, the effect of spinning system is statistically significant on the number of total imperfection values (thin places, thick places and neps) as shown in Table (2). Combed compact yarns have lower total imperfection values than combed conventional ring yarns. The lowest varieties in the number of total imperfection values were Giza 86 and the highest in the number of total imperfection values Giza 94 in both combed compact yarns and conventional ring yarns.

According to Table (3) results present the interaction effect of spinning system and yarn count $(\mathrm{S} \times \mathrm{C}$ ) is statistically significant. Where we find that ( $\mathrm{Ne}$ 120 's) combed compact yarns and conventional combed ring yarns have higher number of total imperfection (thin places, thick places and neps), while at ( $\mathrm{Ne}$ $80 \mathrm{~s}$ ) have lower number of total imperfection. This result can be explained with the weak control of fibers in coarse yarn due to the increased number of fibers in the yarn cross section.

Results presented in Table (3) compare the number of total imperfection (thin places, thick places and neps) in the yarn count (80's, 100's, and 120's) both combed compact yarns and combed conventional ring yarns from the cotton varieties under study. The results showed that number of total imperfection increased with the increase of the yarn count, and that the most increase in number of total imperfection of the variety was Giza 94 and Giza 92 while lowest varieties in decrease in number of total imperfection was the Giza 86.

Mean values of the number of total imperfection (thin places, thick places and neps) summarized in Table (4) and Figure (4). Where there is difference in the number of total imperfection. The results indicate that, there is a significant effect due to the interaction between spinning system, cotton varieties and yarn count $(\mathrm{S} \times \mathrm{V} \times \mathrm{C})$ on the number of total imperfection in yarn. 


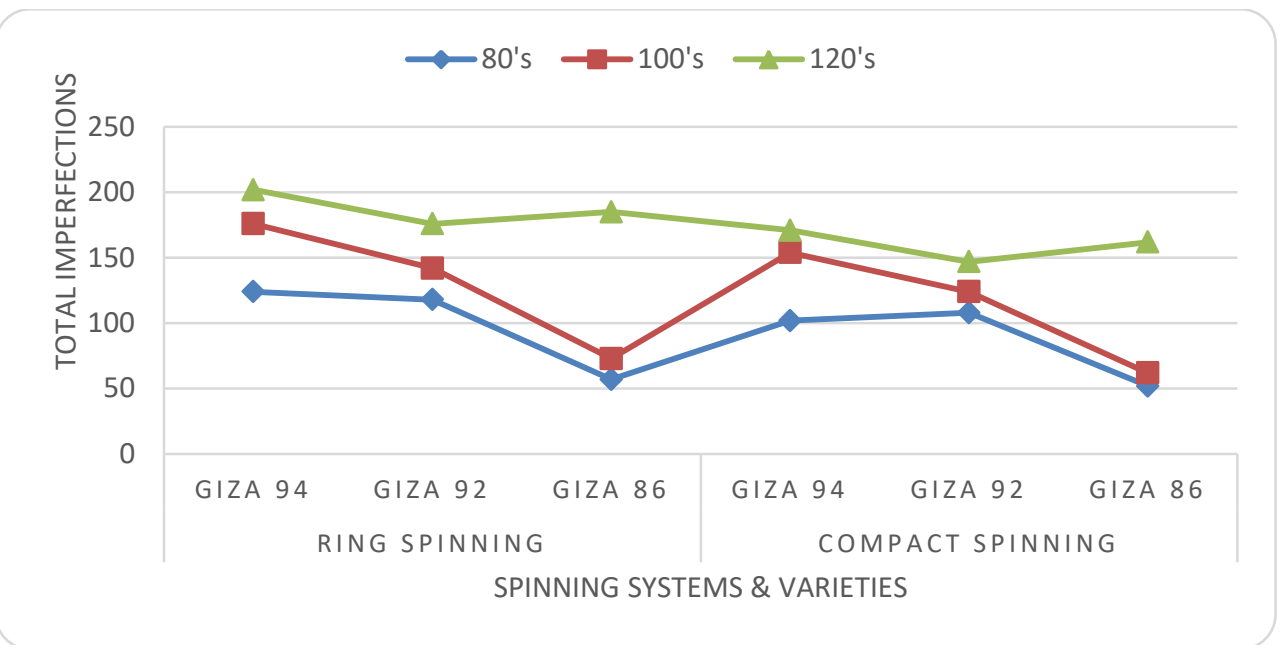

Figure (4). Number of total imperfection (thin, thick places and neps) as affected by spinning systems, cotton varieties and yarn count interaction

\section{Yarn hairiness results:}

The hairiness test results of conventional and compact spun yarns, according to statistical analysis, the effect of spinning system is statistically significant on both combed conventional ring yarns and combed compact yarns hairiness as shown in Table (2). Combed compact yarns have lower hairiness than combed conventional ring yarns. This could be explained by the elimination of spinning triangle in compact yarn spinning system.

Found the hairiness test result the interaction effect of spinning system and yarn count $(\mathrm{S} \times \mathrm{C})$ is statistically significant on combed yarn hairiness as shown in Table (3) and Figure (5). Where there was a significant decrease in the value of the hairiness in both yarn spinning systems of each all yarn count under study.

The interaction effect of spinning system, cotton varieties and yarn count $(\mathrm{S} \times \mathrm{V} \times \mathrm{C})$ is statistically significant on hairiness values of combed yarn. The differences between compact combed and conventional combed yarn hairiness values decreases as the yarn count increases. This shows that the advantage of the compact spinning system on combed yarn hairiness property is more noticeable at higher yarn count levels. This is evident from Tables ( 3 and 4 ) and Figure (5). 


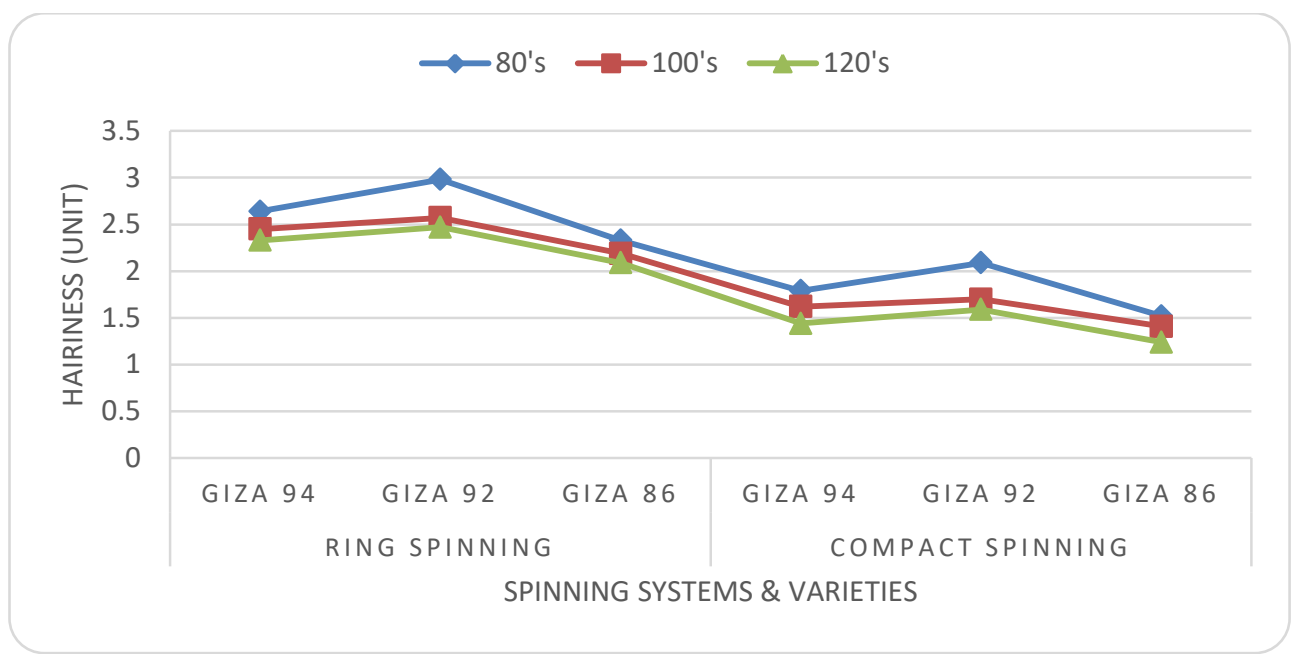

Figure (5). Yarn hairiness index as affected by spinning systems, cotton varieties and yarn count interaction

These results were in the same trend with those of Krifa and Ethridge (2003), Cheng and Yu (2003) and Altas and Kadoğlu (2012) indicated that compact spun yarn strength was found to be higher by about $15 \%$ and elongation at break by about $20 \%$. Furthermore, the yarn hairiness becomes $70 \%$ less, and the coefficient of variation of the yarn was lower by $15 \%$ than that for the equivalent ring spun yarn, as well as, (lqbal, 2018), stated that compact yarns are claimed to be stronger and less hairy dye to the improved fiber binding, and have better yarn elongation and yarn irregularity values compared with conventional ring yarn.

\section{CONCLUSION}

In this study compared between the properties of combed yarns produced with conventional ring and compact yarn spinning systems, the results showed that the compact spinning surpassed than conventional ring spinning in yarn traits e. j. lea count strength product (LCSP), single yarn strength (cN/tex), elongation (\%), CV\%, thin and thick places, neps, total imperfections and yarn hairiness.

As well as cotton variety Giza 92 recorded the desirable values for lea count strength product (LCSP), single yarn strength (cN/tex) and CV\%. On the other side Giza 86 recorded the better values for thin and thick places, neps, total imperfections and yarn hairiness. Yarn count Ne 80 's gave the desirable values for above studied traits.

The first order and second order interaction were significant differences for all studied traits. From the previous results it could be say that cotton variety Giza 92 under both compact spinning and Ne 80's recorded the best values for the studied characters.

In general, the properties of combed yarns produced with compact spinning system are better than those with conventional ring spinning system, compact yarns were having better tensile properties, elongation ratio and lower 
hairiness than conventional ring yarns, because of the elimination of spinning triangle in spinning system, and the decrease from the flight of fibers of structure yarn increase and this leads to higher yarn tenacity and elongation ratio. Also, Compact spun combed yarn was to found to have higher evenness and lower number of total imperfection values (thin places, thick places and neps). If the evenness property of compact yarn can be improved, it will have a potential for improving quality and profitability of cotton yarn manufacturing.

\section{REFERENCES}

Altas, S. and H. Kadoğlu (2012). Comparison of conventional ring, mechanical compact and pneumatic compact yarn spinning systems. J. of Engineered Fibres and Fabrics, 7(1): 87-100.

Artzt, P. (2000). The special structure of compact yarns - advantages in downstream processing. Reprinted from the Proceedings of the Beltwide Cotton Conference Volume 1:798-803 National Cotton Council, Memphis TN.

ASTM (1991). American Society for Testing and Materials. Standards of textile testing and materials, Philadelphia, Pa. (D-5867-05), (D-2256-80), (D1425-81). USA.

Basal, G. and W. Oxenham (2006). Comparison of properties and structures of compact and conventional spun yarns. Tex. Res. J., 76(7): 567-575.

Cheng, K.P.S. and C. Yu (2003). A study of compact spun yarns, Tex. Res. J., 4: 345-349.

El-Sayed, M. A. M. and H. Suzan Sanad (2007). The impact of new spinning technologies on the Egyptian cottons. Autex Res. J., 8 (4):231-238.

Iqbal, S. F. (2018). Influence of yarn structure produced in different spinning systems on the properties of yarn. Int. J. Applied Res., 4(4): 172-176.

Jayavarthanavelu, D. (2006). Compact spinning system-lakshmi RoCoS 1.14, Technical Newsletter Text. Machinery Division, 12:2-4.

Kampen, W. (2000). The advantages of condensed spinning, Melliand English, 4:58-59.

Krause, H. and H. Soliman (1991). Investigation of the strength of the spinning triangle in ring spinning. Melliand Text. berichte, 72:499-506.

Krifa, M. and D. Ethridge (2003). Compact ring yarn: an examination of some production Issues. Text. topics, 2:2-8.

Ömeroğlu, S. and S. Ülkü, (2007). An investigation about tensile strength, pilling and abrasion properties of woven fabrics made from conventional and compact ring-spun yarns. Fibers and Text. in Eastern Europe, 15(1): 39-42.

Sanad, Suzan H., H. E. M. Mahmoud and M. A. M. EL-Sayed (2011). Production of carded compact cotton yarn of comparable quality to the comped conventional ring yarn. J. Agric. Res., 89 (1):203-212.

Staler, H. (1995). A new generation of ring Spun yarns. Melliand Textile berichtee, 76(3): E29-E31.

Staler, H. (2000). New spinning process comfort spin. Melliand International, 6: $1-22$.

Wu, H., M. Chen, W. Wang, K. Lai and B. Ma (2009). The structure of compact yarn. Tex. Res. J., 79 (9): 810-814. 


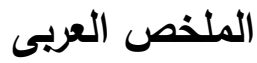 \\ مقارنة بين الغزل الحلقى التقليدي والغزل المدمج لبعض أصناف القطن المصري \\ محمد نجيب محمد البنا'، محمد أحمد عبد الجواد نصار'، محمد عبد الرحمن محمد نجمَ'،

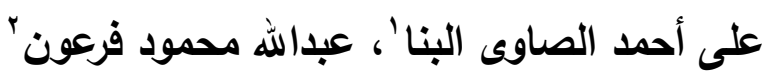 \\ ا ـ قسم الإنتاج النباتى - كلية الزراعة سابا باشا - جامعة الإسكندرية \\ r. معهد بحوث القطن - مركز البحوث الزراعية - الجيزة}

أجريت هذه الدراسة بقسم الإنتاج النباتي بكلية الزراعة (سابا - باشا) جامعة الإسكندرية خلال موسم 19

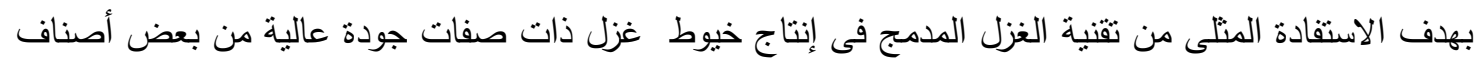
القطن المصرى التجارية ومقارنة صفات هذه الخيوط بمثلتها المنتجة من الغزل الحلقى التقليدى لإمكانية تحسين

جودة الخيوط المنتجة باستخدام تقنيات الغزل الحديثة.

تم إنتاج خيوط ممشطة على نظام الغزل المدمج ونظام الغزل الحلقى التقليدى لثلاثة أصناف من القطن المصري

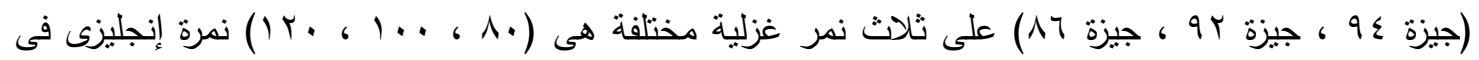

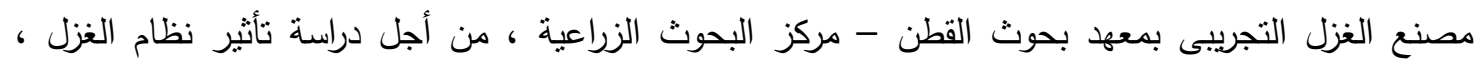
وأصناف القطن ، ونمر الغزل ، والتفاعل بينهم على خصائص الغزول المشطة المنتجة. وقد أوضحت النتائج تفوق نظام الغزل المدمج على نظام الغزل الحلقى التقليدي في صفات خيوط الغزل المنتجة حيث أعطت خيوط الغزل المدمج قيما أقل فى التشعير وقيم منانة أعلى سواء منانة الثلة أو متانة الخيط المفرد ونسبة استطالة أعلى من خيوط الغزل الحلقى التقليدى وربما يرجع السبب وراء انخفاض التتعير في خيوط الغزل هئل المدمج وزيادة منانتة الى تقليل منلث الغزل في نظام الغزل المدمج.

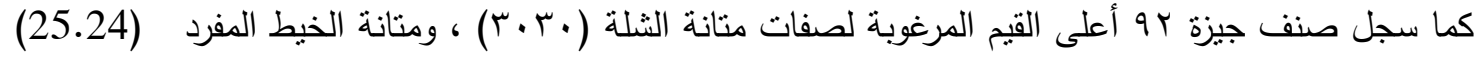

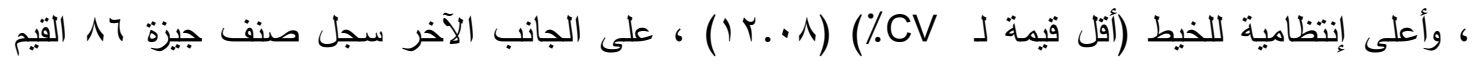

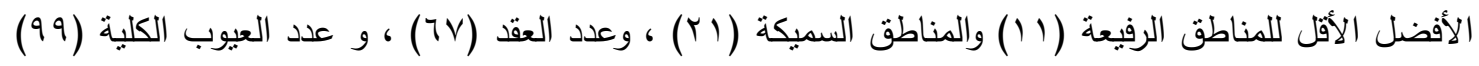
، والتشعير للخيط (·^. (l) ، كما أعطت نمرة الغزل ( Ne 80's ) القيم المرغوب فيها للصفات المدروسة.

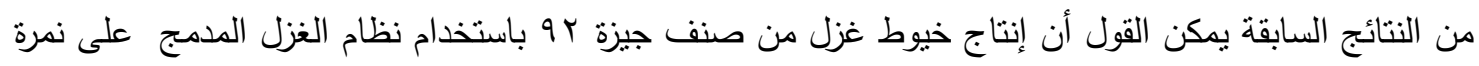
غزل ( Ne 80's ) يعطى النتائج الأفضل والمرغوبة لصفات الخيوط المنتجة. من خلال هذه الدراسة يتضح إمكانية إنتاج خيوط ممشطة عالية الجودة باستخدام نظام الغزل المدمج مقارنة بتلك المنتجة باستخدام الغزل الحلقى التقليدى لأصناف القطن المصرية مما يعطى مؤشرا لإمكانية إنتاج خيوط الغزل المدمج من كافة الأصناف المصرية والهجن المبشرة الجديدة. 